\title{
Adhesion of hollow calcium-deficient hydroxyapatite microspheres onto titanium
}

\author{
Isao KIMURA ${ }^{1}$, Mitsugu KANATANI ${ }^{2}$ and Kouichi WATANABE ${ }^{2}$ \\ ${ }^{1}$ Department of Chemical Engineering, Faculty of Engineering, Niigata University, 8050, Ikarashi 2-no-cho, Nishi-ku, Niigata 950-2181, Japan \\ ${ }^{2}$ Division of Biomaterial Science, Graduate School of Medical and Dental Sciences, Niigata University, 2-5274, Gakko-cho, Chuo-ku, Niigata 951- \\ 8514, Japan \\ Corresponding author, Isao KIMURA; E-mail: ikim@eng.niigata-u.ac.jp
}

\begin{abstract}
Microcapsules containing active drugs, e.g. an antimicrobial agent, with a hydroxyapatite (HAp) shell are expected to prevent infection and to improve osseointegration simultaneously when used as implant materials. This study was conducted to investigate the effects of etching with hydrofluoric acid (HF) to change the surface morphology of titanium in cases of adhesion of calcium-deficient HAp microspheres onto titanium. The microspheres were mixed with Hanks' balanced salt solution (HBSS) to prepare slurry; the slurry was then put between two titanium disks that had been etched and kept soaking in HBSS for seven days at $310 \mathrm{~K}$. The coverage and the degree of adherence of the microsphere were evaluated using electron probe microanalysis. The etching at an HF concentration of $0.10 \mathrm{~mol} / \mathrm{L}$ caused the greatest adhesion. Changing the temperature between 303 and $323 \mathrm{~K}$ showed a tendency for the degree of adherence to increase.
\end{abstract}

Keywords: Hydroxyapatite, Titanium, Microsphere

Received Mar 10, 2009: Accepted Jul 16, 2009

\section{INTRODUCTION}

Antimicrobial agents are applied for preventing infection in cases of implantation. In an oral environment, plenty of saliva and blood is available to sweep away the agents. Therefore, the effect from one administration is expected to be insufficient. It is most desirable that the agent be as persistent as possible. In areas such as pharmacy, engineering, agriculture, and so on, microcapsules are often used to achieve a release over a long period. Microcapsules have been developed for the controlled release of antimicrobial agents ${ }^{1-4}$.

Polymers and organic materials that are biodegradable or digestible are generally used as shell materials of microcapsules used for applications in the oral cavity ${ }^{4-6)}$. They are absorbed in the body over time to contribute to osseointegration or bone conduction if hydroxyapatite (HAp) is used as a shell material. A titanium implant coated with microcapsules containing an antimicrobial agent with a shell made of HAp is expected to be effective for more rapid fixing. For realizing such a material, it is necessary first to obtain fundamental knowledge of the preparation of microcapsules with an HAp shell and their adhesion onto titanium.

One of the authors found that HAp can be synthesized through interfacial reaction in a multiple emulsion $^{7}$. Moreover, hollow HAp microspheres were prepared through investigation of materials and operative conditions ${ }^{8}$. These microspheres are assemblages of calcium-deficient HAp nanoparticles, of which high absorbability into the body is expected. Using TEM observation, they have been recognized as hollow microspheres comprising acicular particles of about $20 \mathrm{~nm}$ on the short axis with a 2.5-10 aspect ratio. Consequently, the space inside the microsphere is inferred to be useful for loading an antimicrobial agent, therefore supporting its application to the microcapsule described above.

The adhesion of HAp particles onto titanium has been reported in relation to the precipitation and coating mainly in the form of nanoparticles and films ${ }^{9 \cdot 14}$. A study on the adhesion of particles or micrometer-size microspheres in stoichiometric or calcium-deficient HAp materials cannot be found in the relevant literature. This study was conducted with the aim of confirming whether the adhesion of hollow calcium-deficient HAp microspheres onto titanium is possible. Titanium was etched with hydrofluoric acid (HF) to change the surface morphology. This study was intended to investigate how the adhesion was affected by the HF concentration in etching. Hank's balanced salt solution (HBSS) was adopted for a medium during the adhesion process as a reliable, well-known buffer solution.

The authors have expected Ostwald ripening as a mechanism of adhesion. In addition, temperature swinging was attempted to improve the adhesion with the aim of using the difference in solubility at different temperatures.

\section{MATERIALS AND METHOD}

Preparation of hydroxyapatite microspheres Hollow calcium-deficient HAp microspheres were prepared using the same method described in a previous paper ${ }^{8}$ : the interfacial reaction was conducted, in which aqueous solutions of potassium hydrogen phosphate $\left(\mathrm{K}_{2} \mathrm{HPO}_{4}\right)$ (special grade, Wako Pure Chemical Industries, Ltd., Osaka, Japan) and calcium nitrate tetrahydrate $\left(\mathrm{Ca}\left(\mathrm{NO}_{3}\right)_{2} \bullet 4 \mathrm{H}_{2} \mathrm{O}\right)$ (special grade, 
Wako Pure Chemical Industries, Ltd., Osaka, Japan) were used respectively as the inner and outer phases. The preparation conditions are presented in Table 1.

\section{Preparation of titanium disks}

A titanium rod of $20 \mathrm{~mm}$ diameter (JIS class 2, Nilaco Corp., Tokyo, Japan) was cut into 3-mm-thick disks. They were wet polished with silicon carbide waterproof abrasive papers of $40,25,14$, and $11.5 \mu \mathrm{m}$ grit in turn. Finally, a colloidal silica suspension of $60 \mathrm{~nm}$ in particle size (Mastermet, Buehler Ltd., Lake Bluff, USA) was used as the finish to obtain a mirror surface. Each disk was immersed in HF (special grade, Wako Pure Chemical Industries, Ltd., Osaka, Japan) for 24 min at concentrations of $0.010-0.50 \mathrm{~mol} / \mathrm{L}$ and a temperature of $303 \mathrm{~K}$. Each was washed with a distilled water stream from a washing bottle, sprayed with hydrochlorofluorocarbon (HCFC) (Aeroduster ZC34, Engineer Inc., Osaka, Japan) to remove the moisture quickly; then each was stored in a desiccator.
Adhesion of hydroxyapatite microspheres onto a titanium disk

A 12-well microplate made of polystyrene (\#3815-012, Asahi Techno Glass Corp., Funabashi, Japan), which has an array of holes of $22 \mathrm{~mm}$ diameter and $17 \mathrm{~mm}$ depth, was used as a treatment vessel. Slurry was prepared by mixing $0.60 \mathrm{~g}$ of the microsphere with $3.00 \mathrm{~g}$ of HBSS without calcium and magnesium ions (BioWhittaker Inc., Walkersville, USA). A polytetrafluoroethylene (PTFE) disk of $3 \mathrm{~mm}$ thickness was placed in a hole of the microplate, and the titanium disk was put on it. Then, $1.6 \mathrm{~g}$ of the slurry and another titanium disk were put on them. Three PTFE rods were inserted into a gap separating titanium disks and the hole wall so that the disks did not move. All holes were filled with HBSS, and the microplate was covered with the lid, followed by sealing. This was heated for $168 \mathrm{~h}$ in a water bath according to the temperature profiles portrayed in Fig. 1. The temperature was maintained at $310 \mathrm{~K}$ in profile $\mathrm{A}$. In profile $\mathrm{B}$, the temperature was kept at $323 \mathrm{~K}$ for $48 \mathrm{~h}$ and then repeatedly swung between 303 and $323 \mathrm{~K}$ for

Table 1 Experimental condition for preparing hydroxyapatite microspheres

\begin{tabular}{ll}
\hline Inner aqueous phase & $0.30 \mathrm{~mol} / \mathrm{kg} \mathrm{K}_{2} \mathrm{HPO}_{4}$ \\
Outer aqueous phase & $0.50 \mathrm{~mol} / \mathrm{kg} \mathrm{Ca}\left(\mathrm{NO}_{3}\right)_{2}$ \\
Oil phase & Cyclohexane \\
Surfactant in the oil phase & 5.0 mass\% Sorbitan monooleate \\
Surfactant in the outer aqueous phase & 0.50 mass\% Polyoxyethylene (20) sorbitan monolaurate \\
pH of the inner aqueous phase & 12.0 \\
Preparation temperature & $323 \mathrm{~K}$ \\
Preparation duration & $24 \mathrm{~h}$ \\
\hline
\end{tabular}

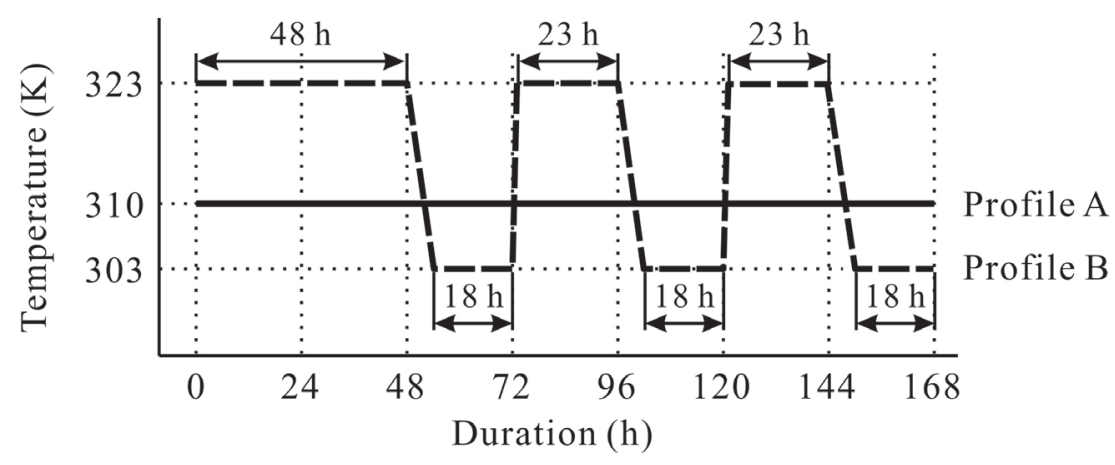

Fig. 1 Temperature profiles during the adhering process. The temperature was kept at $310 \mathrm{~K}$ in profile $\mathrm{A}$ and changed repeatedly between at 303 and $323 \mathrm{~K}$ in profile B. 


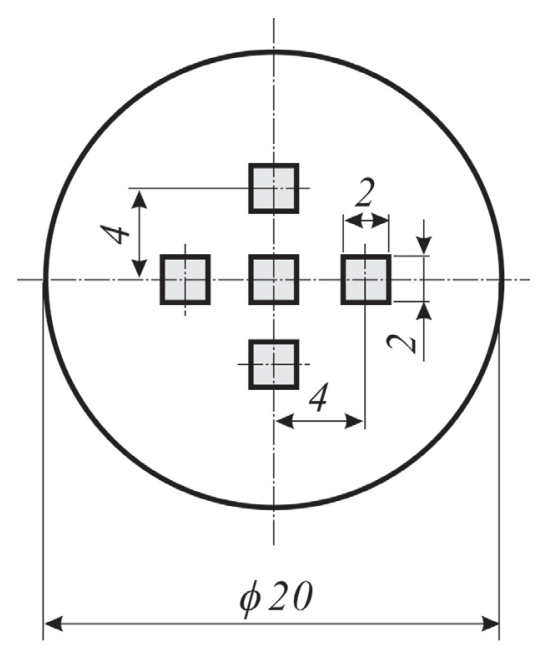

(unit: $\mathrm{mm}$ )

Fig. 2 Analyzed areas in EPMA.

$24 \mathrm{~h}$ each. Under this condition, it took respectively $6 \mathrm{~h}$ and $1 \mathrm{~h}$ on cooling from 323 to $303 \mathrm{~K}$ and on heating from 303 to $323 \mathrm{~K}$. This process will be designated as the 'adhesion process' hereafter.

The specimen was roughly washed with a distilled water stream from a washing bottle. Then it was put in water in a beaker, in which the water was stirred at a linear rate of about $2 \mathrm{~m} / \mathrm{s}$ on the specimen surface, for 30 min to remove HAp microspheres that had loosely adhered. This process will be designated hereafter as the 'washing process'. Finally, it was flushed with a washing bottle, sprayed with HCFC, and kept in a desiccator.

\section{Characterization}

The surface morphology of the etched titanium disks and the adhesion features of the HAp microspheres were observed using a scanning electron microscope (SEM) (JSM-5800, JEOL, Tokyo, Japan). The coverage was defined as the ratio of the projected area of the microspheres to the surface of a titanium disk. It was evaluated from the mapping analysis using an electron probe microanalyzer (EPMA) (EPMA-8705, Shimadzu Corp., Kyoto, Japan) for CaKo at an acceleration voltage of $15 \mathrm{kV}$ and a specimen current of $0.3 \mu \mathrm{A}$. The analysis was conducted in five areas of $2 \mathrm{~mm}$ square, arrayed symmetrically around the center, as portrayed in Fig. 2. The characteristic X-ray image was binarized using a threshold value, which was determined so that the size on the binarized image became $1 \mu \mathrm{m}$ for a particle recognized as $1 \mu \mathrm{m}$ on the secondary electron image at the same site. The degree of adherence was evaluated using the mean intensity of X-ray count of $\mathrm{CaK} \alpha$ for each specimen after subtracting that for the background on the assumption that the intensity is proportional to the HAp amount.

\section{Statistical Analyses}

The significant difference between two data related to the coverage and the degree of adherence was examined using a $t$-distribution test for the difference between two average pairs. Welch's method was used when their variances were not found to be equal. The assessed significance corresponding to the significance level is denoted, respectively, with significance symbols: $*$, **, and ${ }^{* * *}$ for $p<0.05,0.01$, and 0.001 .

\section{RESULTS}

\section{Surface state of the etched titanium disks}

Figure 3 depicts that the surface morphology of the titanium disks was changed remarkably by $\mathrm{HF}$ concentration. The surface of the non-etched specimen (a) is confirmed to be completely smooth. By etching at $0.010 \mathrm{~mol} / \mathrm{L}$ (b), pits smaller than $0.3 \mu \mathrm{m}$ were produced over the entire surface. They are apparently arranged within each grain. The pits became larger at higher HF concentrations (c-d), e.g. approximately $0.6 \mu \mathrm{m}$ at 0.10 $\mathrm{mol} / \mathrm{L}$. Hillocks are observed as peaked walls left without being dissolved. Dimples of approximately 1-2 $\mu \mathrm{m}$ diameter can be recognized in places like the circled area. At much higher concentrations (e)-(f), the surface was conversely flattened.

\section{Adhesion of the HAp microspheres}

Figure 4 portrays a scanning electron micrograph of the HAp microspheres prepared using the interfacial reaction. They can be recognized as discrete microspheres of around $1 \mu \mathrm{m}$ diameter.

Figure 5 presents a typical example of the adhering state of the microspheres on a titanium disk etched with $\mathrm{HF}$ at a concentration of $0.10 \mathrm{~mol} / \mathrm{L}$. The micrograph at low magnification (a) demonstrates that the titanium disk surface is covered nonuniformly with the microspheres, which seem to assemble as islets. Compared with the microspheres before the adhering treatment portrayed in Fig. 4, the boundaries between microspheres are unclear on the micrograph at a higher magnification (b).

Figure 6 portrays effects of the HF concentration on the coverage. The microspheres adhered only slightly onto the non-etched specimen, but the adhesion was promoted by etching. The coverage was increased at higher $\mathrm{HF}$ concentrations from $4.4 \%$ at $0.010 \mathrm{~mol} / \mathrm{L}$ to $66 \%$ at $0.10 \mathrm{~mol} / \mathrm{L}$. At much higher $\mathrm{HF}$ concentrations, the coverage was decreased. Significant differences were found, respectively, between the value of the coverage at $0.10 \mathrm{~mol} / \mathrm{L}$ and those at 0.050 and $0.20 \mathrm{~mol} / \mathrm{L}(p<0.001)$.

The effects of the HF concentration on the degree of adherence are portrayed in Fig. 7. This graph reveals a maximum at an $\mathrm{HF}$ concentration of $0.10 \mathrm{~mol} / \mathrm{L}$, in addition to significant differences around this concentration as well as a result of the coverage.

Effects of temperature profiles

Figure 8 portrays effects of the temperature swinging 
on the coverage. The coverage was larger in profile $\mathrm{B}$ than that in profile $\mathrm{A}$ over the wide $\mathrm{HF}$ concentration range of $0.050-0.20 \mathrm{~mol} / \mathrm{L}$. However, a significant difference was not recognized. Regarding the degree of adherence, significant improvement attributable to the temperature swinging method was recognized at 0.025 , 0.050 , and $0.20 \mathrm{~mol} / \mathrm{L}$, as shown in Fig. 9, but not at $0.10 \mathrm{~mol} / \mathrm{L}$.
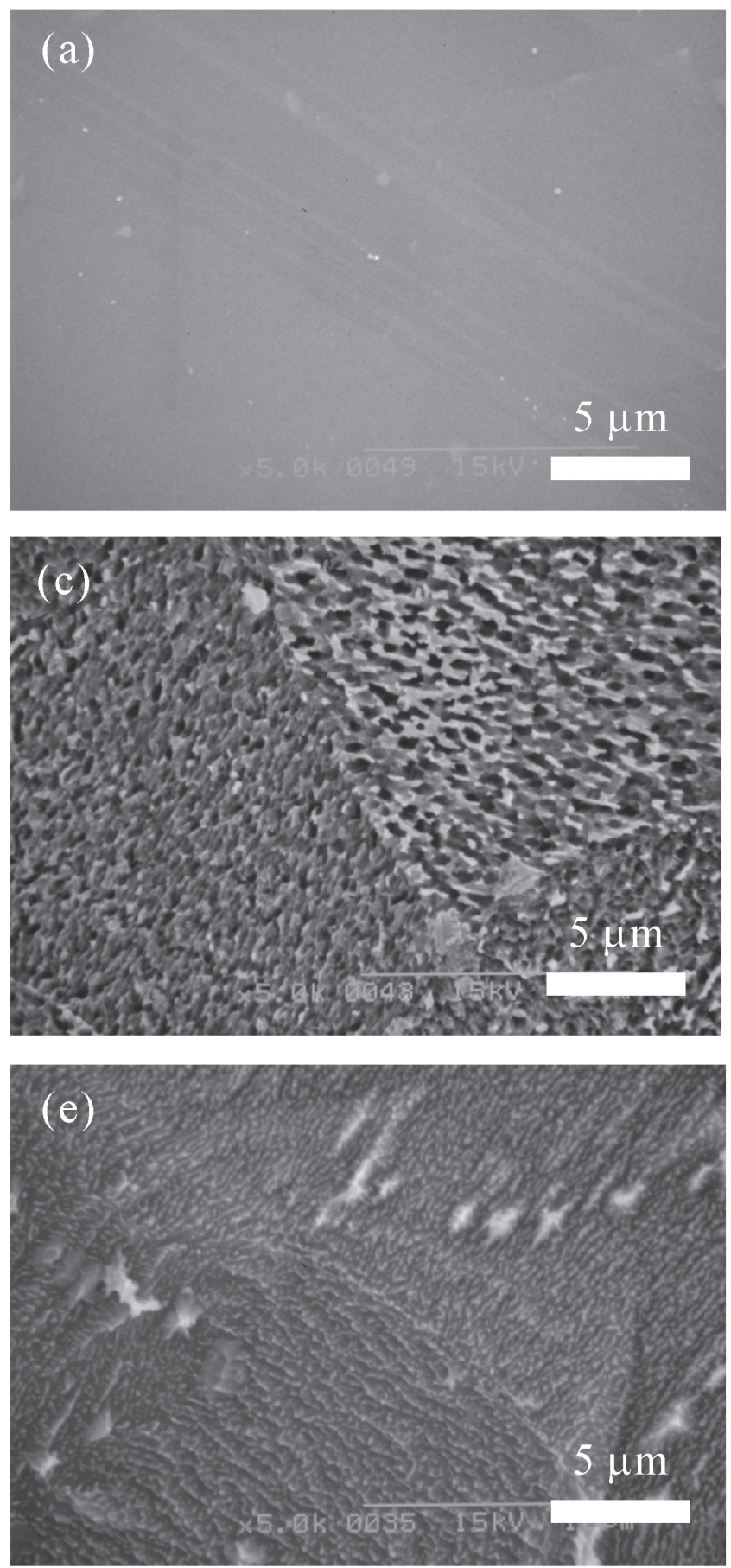

\section{DISCUSSION}

To elucidate the changes in the surface morphology of titanium by etching, Fig. 10 was illustrated from SEM micrographs including results depicted in Fig. 3. At a low $\mathrm{HF}$ concentration (a), dissolution proceeds to form shallow pits, which may reveal the most stable crystalline plane. Their patterns differ from grains
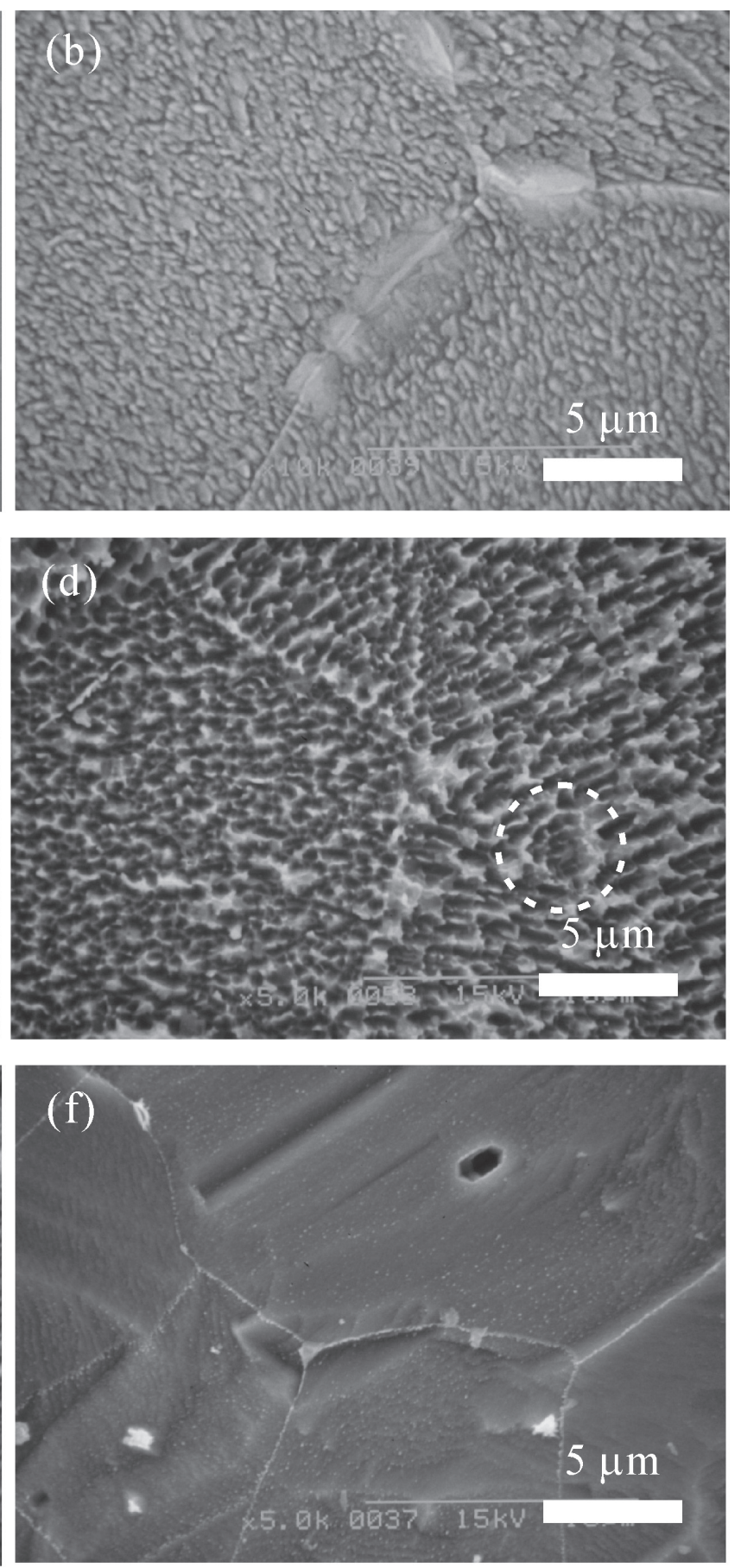

Fig. 3 Scanning electron micrographs of the titanium disks: (a) Non-etched; (b)-(f) Etched respectively with HF at concentrations of (b) 0.010, (c) 0.025, (d) 0.10, (e) 0.20, and (f) $0.50 \mathrm{~mol} / \mathrm{L}$. 


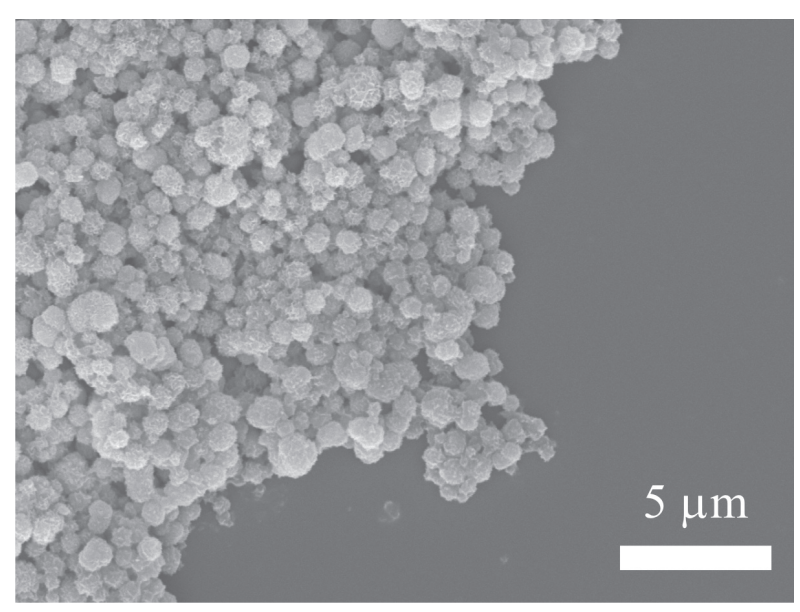

Fig. 4 Scanning electron micrograph of HAp microspheres prepared using the interfacial reaction in a multiple emulsion.
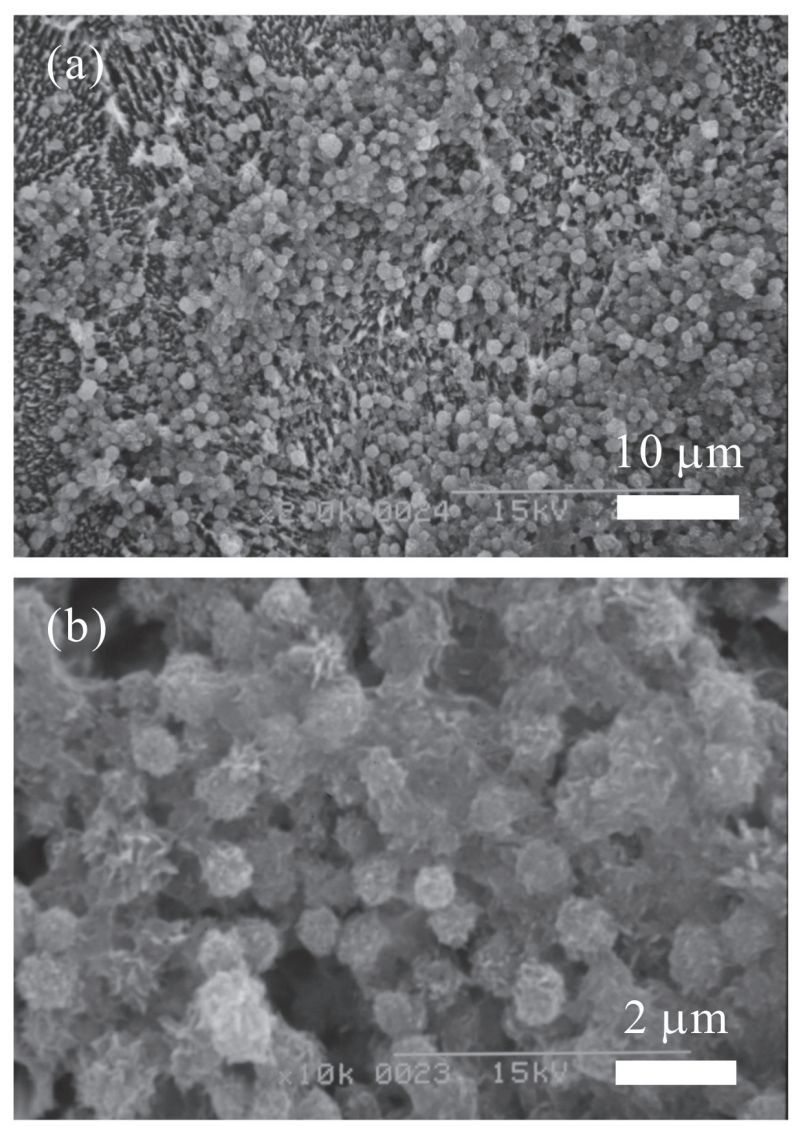

Fig. 5 Scanning electron micrographs of the HAp microspheres adhering on the titanium disk etched with $\mathrm{HF}$ at a concentration of $0.10 \mathrm{~mol} / \mathrm{L}$; (b) is a higher magnification of (a).

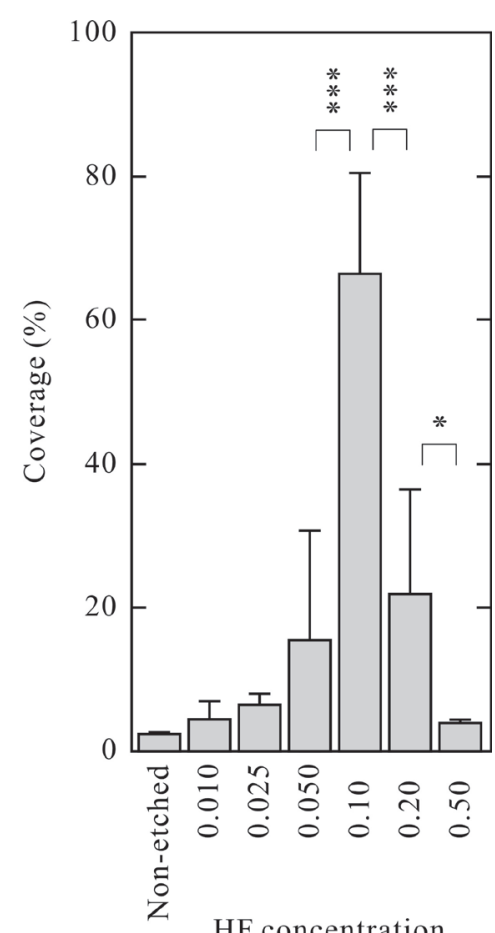

HF concentration

$(\mathrm{mol} / \mathrm{L})$

Fig. 6 Effects of HF concentration on the coverage.

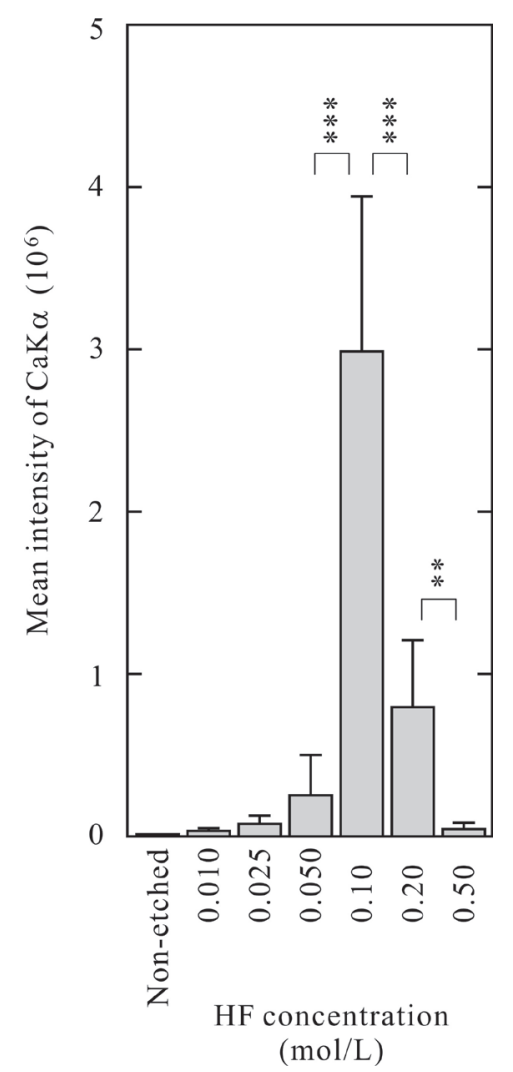

Fig. 7 Effects of $\mathrm{HF}$ concentration on the degree of adherence. 


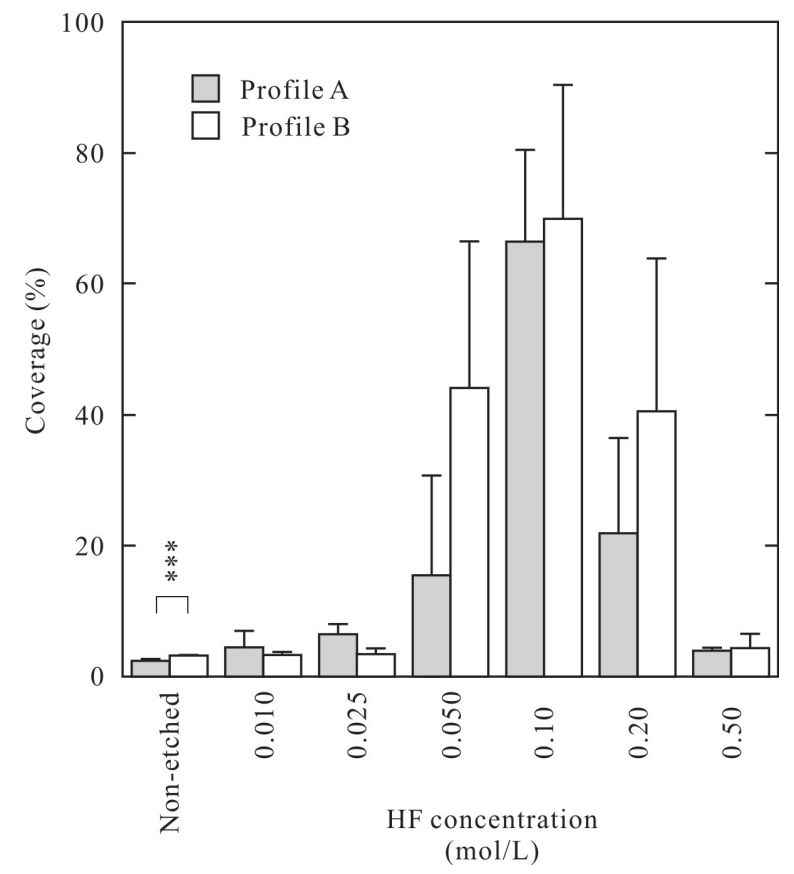

Fig. 8 Effects of temperature profiles on the coverage.

because of the randomness of direction appearing in the polished surface. At a medium HF concentration (b), the surface is eroded deeply to form hillocks. Some hillocks with different height happen to form a dimple. The tip of a hillock is dissolved preferentially by $\mathrm{HF}$ at a high concentration (c), so that the surface is flattened.

Most microspheres which had not adhered during the adhesion process were washed away by a water stream from a washing bottle. Furthermore, some microspheres were washed away during the subsequent washing process in a beaker. Figure 4 portrays a rough surface that was typical of the microspheres prepared in this study. Powders having such morphology are generally poor in fluidity and are prone to agglomerate. When the microspheres piled up during the adhering process, they had a large projection area against a water stream in the washing process. Intensive drag force is therefore exerted on the agglomerates to be detached. Conversely, for the agglomerates that had been stacked low, the exerted drag force must be sufficiently small that they can remain adherent. The microspheres presented in Fig. 5(a) remained adherent during the washing process. Therefore, it can be thought that the adhesion is of certain strength sufficient to be handled in practical applications. Figure 5(b) suggests that the adhesion was promoted through dissolution and precipitation of the microspheres.

Figure 6 shows that the largest value of the coverage was $66 \%$. Nishimura et al. reported that the

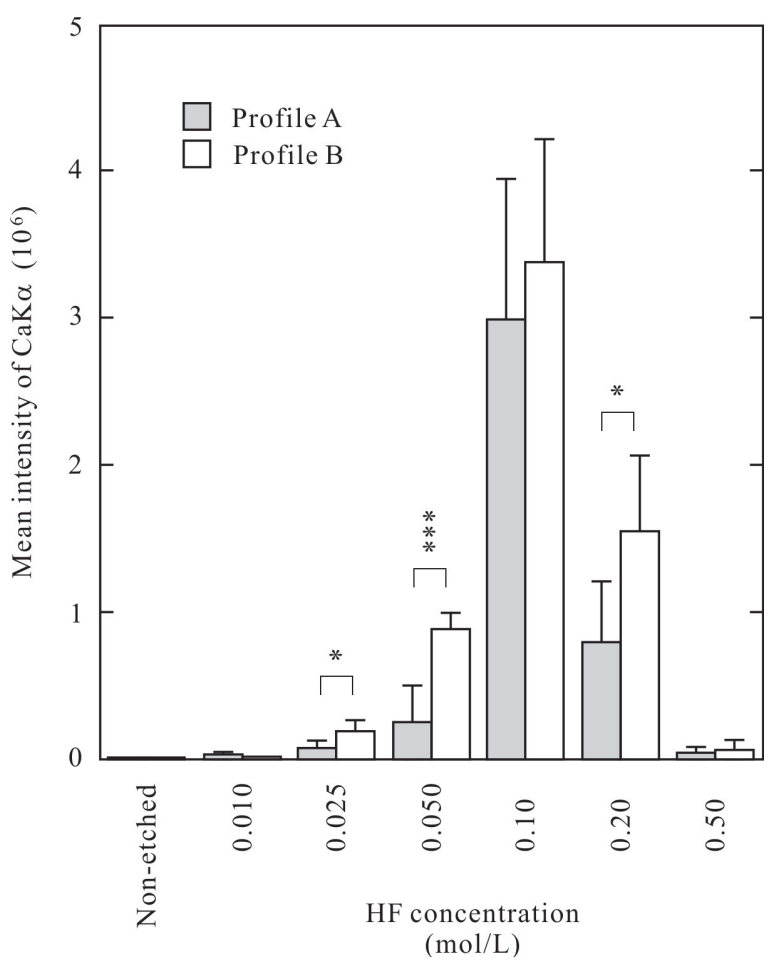

Fig. 9 Effects of temperature profiles on the degree of adherence.
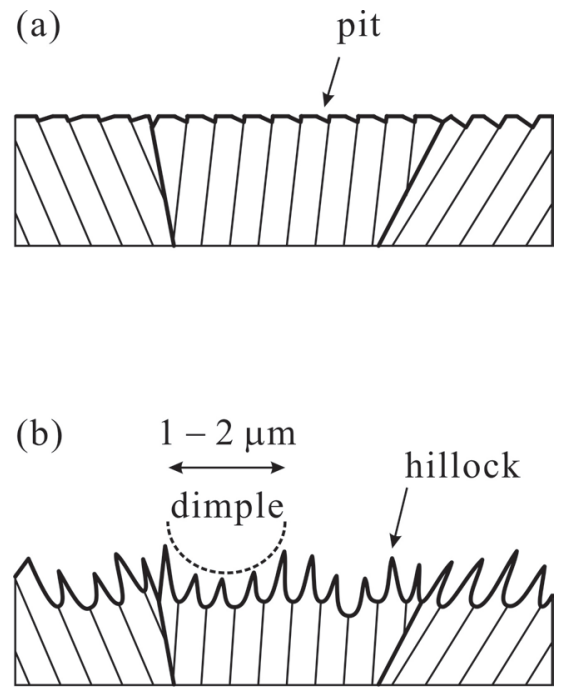

(c)

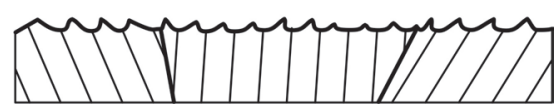

Fig. 10 Schematic illustration of the change in the surface morphology of titanium by etching at (a) a low, (b) a medium, and (c) a high HF concentration. 
coverage of about 50\% with HAp nanoparticles is sufficient to accelerate osseointegration ${ }^{14)}$. Consequently, the specimen prepared in this study is expected to be effective for improving the biological affinity.

As presented for comparison in Figs. 6 and 7, the results show that the degree of adherence was altered more remarkably than the coverage. In other words, the increase in volume of the microspheres which adhered was greater than the increase in area: the adhesion of a microsphere onto microspheres which had already adhered occurred more readily than that onto titanium. The adhesion onto microspheres indicates the progression of agglomeration. As described above, excessive agglomeration causes the microspheres to be detached. It might be difficult to promote adhesion further by controlling the etching conditions.

It cannot be concluded from Fig. 8 that the coverage is affected by the temperature swinging. However, Fig. 9 depicts the improvement in the degree of adherence achieved using such an approach, except for the etching at $0.10 \mathrm{~mol} / \mathrm{L}$. These results suggest that the temperature swinging is effective for promoting adhesion among microspheres. For the specimen etched at $0.10 \mathrm{~mol} / \mathrm{L}$, piled agglomerates should be removed easily during the washing process.

Based on the description given above, the adhering mechanism of the HAp microspheres onto titanium in this study will be discussed. In fact, HAp can join metallic titanium with a titanium oxide layer. The titanium disk was finished in this study as a mirror to show a silvery, metallic luster. It is known that the thickness of oxide film grown on pure titanium at room temperature is of the order of 3 to $7 \mathrm{~nm}^{15)}$. Even if the microspheres of $1 \mu \mathrm{m}$ diameter adhered strongly to the oxide layer of such thickness, it is unlikely that they would be able to continue adhering while withstanding shear stress during the washing process. The principal mechanism should be a simple physicochemical process.

It is easy to recognize that the etching of titanium can affect the adhesion of the microspheres onto titanium, and not the adhesion between the microspheres. The microspheres should stack on the other microspheres to the same extent independent of the etching condition. Therefore, the shear stress by water stream applied to the microsphere layer is the same for any specimen.

The relationship between the etching condition and the degree of adherence can be discussed as follows. When the surface of titanium is completely smooth, it contacts a microsphere just at a point. The shear stress is concentrated at the contact point, so that it breaks readily. As some solid is precipitated around the contact point, the contact is extended from a point to an area. It causes the increase in the resistance against the shear. If the microspheres which have adhered onto titanium are linked through the other microspheres, even though each contact is a point, the apparent contact area increases rapidly. When dimples with size comparable to that of the HAp microspheres are formed on the titanium disk surface, the microspheres can be held temporarily in such dimples. Such a microsphere can make contact by its hemispherical area. The constituent ions of HAp dissolved in HBSS are precipitated at the contact as to act as glue. The holding does not occur in dimples which are either too small or too large. The dimples of the size comparable to that of the microsphere are produced at an $\mathrm{HF}$ concentration of $0.10 \mathrm{~mol} / \mathrm{L}$. We are convinced that this is the reason why the adhesion was the greatest for the specimens etched at this concentration.

The improvement of adhesion by temperature swinging can be attributed to the repetition of dissolution and precipitation. Therefore, the adhesion is expected to improve further by optimizing the temperature profile according to the precise data of the HAp microspheres in terms of solubility and dissolution rate.

\section{CONCLUSIONS}

The adhesion of calcium-deficient HAp microspheres prepared using the interfacial reaction onto titanium was attempted; the effects of etching with $\mathrm{HF}$ on adhesion were investigated. The titanium disk surface morphology was altered with $\mathrm{HF}$ at various concentrations; a different degree of adherence was attained. Results showed that the etching at an $\mathrm{HF}$ concentration of $0.10 \mathrm{~mol} / \mathrm{L}$ caused the greatest adhesion. When the temperature was swung during the adhesion process, the degree of adherence was improved. The results of this study proved that, in principle, the HAp microspheres can be made to adhere onto titanium using a simple procedure.

\section{ACKNOWLEDGMENTS}

Part of this study was financially supported by a Grantin-Aid for Scientific Research (C) No. 18560666, the Japan Society for the Promotion of Science and a Grant for Promotion of Niigata University Research Projects. We greatly appreciate Mr. M. Kobayashi, Center for Instrumental Analysis, Niigata University, for his helpful advice and operation of EPMA.

\section{REFERENCES}

1) Kimura I, Tanaka M, Taniguchi M. Preparation of microcapsules containing silver-complex by sol-gel process in reverse suspension. Proceedings of the 6th World Congress of Chemical Engineering, Melbourne, Australia, 2001, pp.1-94.

2) Kromidas L, Perrier E, Flanagan J, Rivero R, Bonnet I. Release of antimicrobial actives from microcapsules by the action of axillary bacteria. Int J Cosmet Sci 2006; 28: 103108.

3) Peng X, Zhang L, Kennedy JF. Release behavior of microspheres from cross-linked N-methylated chitosan encapsulated ofloxacin. Carbohydrate Polym 2006; 65: 288295.

4) Park YJ, Lee JY, Yeom HR, Kim KH, Lee SC, Shim IK, 
Chung CP, Lee SJ. Injectable polysaccharide microcapsules for prolonged release of minocycline for the treatment of periodontitis. Biotechnol Lett 2005; 27: 1761-1766.

5) Bromberg L. Polymeric micelles in oral chemotherapy. J Control Release 2008; 128: 99-112.

6) Nishino S, Kishida A, Yoshizawa H. Morphology control of polylactide microspheres enclosing irinotecan hydrochloride with polylactide based polymer surfactant for reduction of initial burst. Int J Pharm 2007; 330: 32-36.

7) Kimura I. Synthesis of hydroxyapatite by interfacial reaction in a multiple emulsion. Res Lett Mater Sci 2007; 2007: ID71284.

8) Kimura I, Honma T, Riman RE. Preparation of hydroxyapatite microspheres by interfacial reaction in a multiple emulsion. J Ceram Soc Jpn 2007; 115: 888-893.

9) Ban S, Matsuo K, Mizutani N, Hasegawa J. Hydrothermalelectrochemical deposition of calcium phosphates on various metals. Dent Mater J 1999; 18: 259-270.

10) Stoch A, Brozek A, Kmita G, Stoch J, Jastrzebski W, Rakowska A. Electrophoretic coating of hydroxyapatite on titanium implants. J Mol Struct 2001; 596: 191-200.

11) Tamura M, Endo K, Maida T, Ohno H. Hydroxyapatite film coating by thermally induced liquid-phase deposition method for titanium implants. Dent Mater J 2006; 25: 3238.

12) Forsgren J, Svahn F, Jarmar T, Engqvist H. formation and adhesion of biomimetic hydroxyapatite deposited on titanium substrates. Acta Biomater 2007; 3: 980-984.

13) Albayrak O, El-Atwani O, Altintas S. Hydroxyapatite coating on titanium substrate by electrophoretic deposition method: Effects of titanium dioxide inner layer on adhesion strength and hydroxyapatite decomposition. Surf Coat Technol 2008; 202: 2482-2487.

14) Nishimura I, Huang Y, Butz F, Ogawa T, Lin A, Wang CJ. Discrete deposition of hydroxyapatite nanoparticles on a titanium implant with predisposing substrate microtopography accelerated osseointegration. Nanotechnol 2007; 18: 245101.

15) Brunette DM, Tengvall P, Textor M, Thomsen P. Titanium in Medicine. Springer, Berlin, 2001, pp. 181. 